\title{
Amygdala lesions: Differential effects on activity and reactivity to light of rats*
}

\author{
SHERWOOD O. COLE \\ Rutgers University, Camden, New Jersey 08102
}

\begin{abstract}
The activity of amygdala-lesioned rats was compared with that of normal controls and amygdala shams under shuttlebox conditions of total darkness and single-compartment illumination. Although the activity (movement between compartments) of the groups did not differ under either of these conditions, the single-compartment illumination significantly decreased the activity of all three groups. A further analysis of the percentage of test time spent in the illuminated compartment indicated that the amygdala-lesioned animals spent significantly less time there than did the other two groups. The differential effects of the lesions on activity and reactivity to light were discussed in terms of changes in general responsiveness following amygdala damage.
\end{abstract}

Amygdala-lesioned animals demonstrate a different sensitivity from that of nonlesioned animals in a variety of experimental conditions. This may be due to a general alteration in their responsiveness to environmental stimuli (Nauta, 1963). In feeding tests, amygdala lesions decrease the sensitivity of rats to quinine solution (White, 1971) and to reinforcement contingencies (Schwartzbaum, 1960), and increase the sensitivity of rats to the context within which feeding takes place (Sclafani et al, 1970). Although Sclafani et al (1970) found no difference in the activity of amygdala-lesioned rats and controls in a moderately illuminated open-field test, Schwartzbaum and Gay (1964) reported that amygdala lesions increased the activity of rats in a highly illuminated open field. Also, Schwartzbaum (1965) reported that amygdalectomized monkeys were debilitated in the performance of tasks involving vision and proprioception. Although it is difficult to predict the directional effect of amygdala lesions, the change in sensitivity seems to hold over a fairly wide range of behavior.

The present study was undertaken to compare the shuttlebox activity of amygdala-lesioned rats with that of normal controls and amygdala shams and to use such a context to further evaluate the reactivity of lesioned animals to apparatus illumination.

\section{METHOD}

\section{Subjects}

Thirty-six 120-day-old male albino rats of the Charles-River strain, weighing $350-425 \mathrm{~g}$, were used. They were housed in individual plastic cages in a temperature-controlled laboratory

*This research was supported by a faculty fellowship from the Rutgers University Research Council and was conducted while the author was a visiting investigator at the Laboratory of Psychobiology and Psychopharmacology, Rome, Italy, during the academic year of 1971-72. The gracious support of Professors D. Bovet and A. Oliverio during the course of the research and the valuable technical and statistical assistance of $P$. $\mathrm{E}$. Gay in the preparation of this manuscript are gratefully acknowledged. under normal day-night cycles and had ad lib access to Purina Chow and water in the home cages.

\section{Apparatus}

Six standard shuttleboxes, housed in a soundproof walk-in chamber, were used for testing. Each shuttlebox consisted of two $24 \times 21 \mathrm{~cm}$ compartments (A and B), which were separated by a central divider with a $9 \times 9 \mathrm{~cm}$ door, allowing freedom of access to either side. Both compartments were equipped with two 10-W bulbs located in the overhead cover of the box, which provided illumination when appropriate. Contact points under the four corners of the grid floor of the shuttlebox provided a measure of the number of crossings from one compartment to the other, as well as the amount of time spent in each compartment. Such information was automatically recorded on Esterline-Angus tapes for analysis. Neither food nor water was available to $S s$ in the apparatus, and the boxes were covered with black cloth material during testing to eliminate the diffused lighting in the walk-in chamber.

\section{Surgical Procedure}

Animals were assigned randomly to surgical conditions (normal-control, amygdala-sham, amygdala-lesion) to form three equal groups of 12 rats each. In the amygdala-lesion group, bilateral electrolytic lesions (2-mA anodal dc current for $20 \mathrm{sec})$ were produced stereotaxically with monopolar stainless steel electrodes, insulated except at the tip, while the animals were anesthetized with Nembutal sodium $(50 \mathrm{mg} / \mathrm{kg})$. A rectal cathode completed the circuit. Flat-skull stereotaxic coordinates for the amygdala were $-0.5 \mathrm{~mm}$ posterior to bregma, $\pm 4.0 \mathrm{~mm}$ lateral to midline, and $-8.0 \mathrm{~mm}$ vertical depth (Skinner, 1971). The procedure for the amygdala-sham operation was the same as above, except that no current was passed following electrode placement.

\section{Test Procedure}

Initially, animals in all groups were administered three successive 50 -min activity trials in order to establish a reliable baseline level against which to compare the subsequent effectiveness of compartment illumination. These three trials were conducted under conditions of total apparatus darkness on subsequent weeks. On all three trials, two animals from each of the three surgical groups were tested simultaneously, with a total of 12 animals tested on each of 3 successive days during the hours of $3: 00-6: 00$ p.m. The total number of crossings between compartments for each of the three trials and the number of crossings for successive $10-\mathrm{min}$ periods of the $50-\mathrm{min}$ test on 


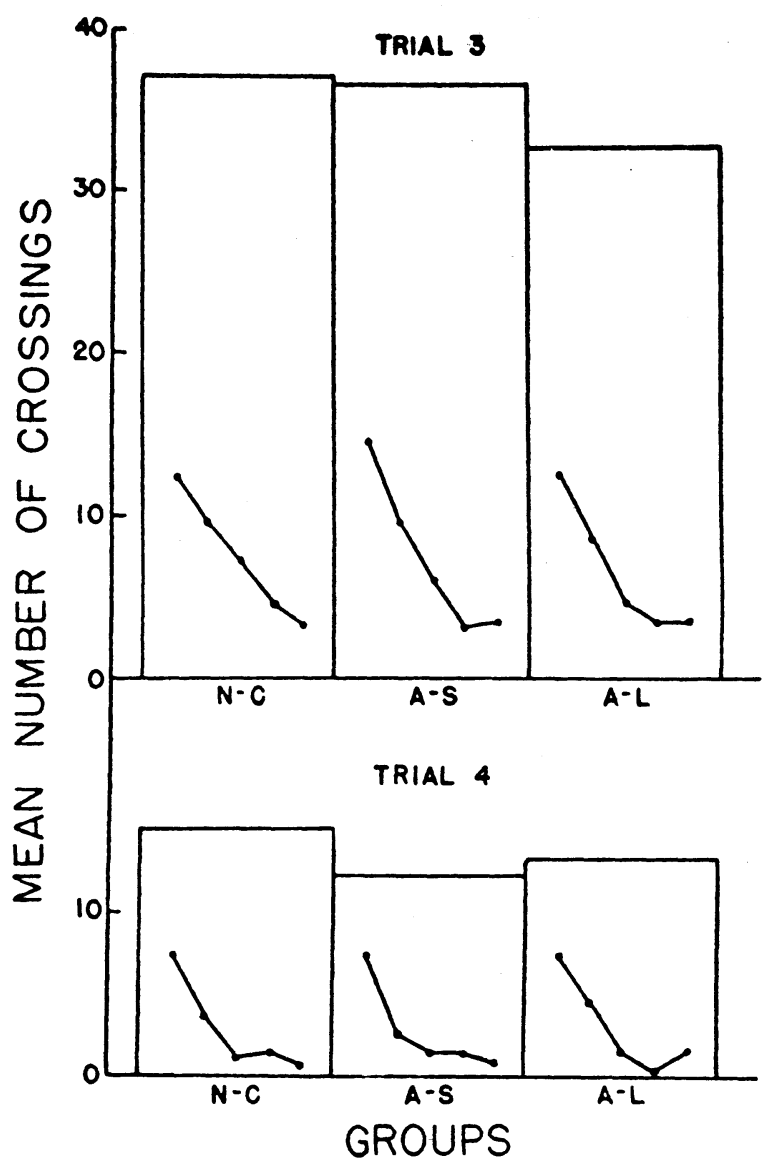

Fig. 1. The total number of crossings and crossings for successive $10-\mathrm{min}$ periods of a 50 -min activity test by normal-control (N-C), amygdala-sham (A-S), and amygdala-lesion (A-L) animals under conditions of total darkness (Trial 3) and single-compartment illumination (Trial 4).

Trial 3 were determined.

On Trial 4 (1 week after Trial 3), all animals in the three groups were administered another 50 -min activity test under conditions of single-compartment illumination. To control for position effect, half of the Ss in each of the groups received illumination in Compartment $A$ and the other half in Compartment B. Animals were tested on the same days of the week during the same hours as in the previous trials. In addition to the total number of crossings between compartments and crossings for successive 10 -min periods of the test being measured, the percentage of total test time spent in the illuminated compartment (measure of reactivity to light) was calculated for each animal in all three groups from the record tapes.

Following completion of the experiment, the amygdala-lesion and amygdala-sham animals were sacrificed and perfused with $37 \%$ formaldehyde solution; the brains were then extracted and sectioned (40 microns) for staining.

\section{RESULTS}

Analysis of mean body weights for the three surgical groups indicated no significant difference on any of the trials. Therefore, the activity data were analyzed without statistical adjustment.

Analysis of activity on Trials 1-3 yielded a significant trials effect only $(F=15.79$, df $=2 / 64, p<.01)$. Further orthogonal comparisons indicated that Trial 1 differed significantly from Trials 2 and 3 combined $(F=$ $30.77, \mathrm{df}=1 / 64 \mathrm{p}<.01$ ), but that Trials 2 and 3 did not themselves differ significantly $(\mathrm{F}<1.00)$. Thus, in spite of the apparatus novelty effect that was apparently present on Trial 1, the absence of a significant difference between Trials 2 and 3 indicated that the activity levels on Trial 3 provided a reliable baseline against which to evaluate the subsequent effectiveness of Trial 4 compartment illumination.

The total number of crossings between compartments and number of crossings for successive 10-min periods on Trials 3 and 4 are summarized in Fig. 1. Analysis of these data yielded no significant difference in the total activity of the three surgical groups under Trial 3 conditions of total apparatus darkness $(F<1.00)$ or under Trial 4 conditions of single-compartment illumination $(F<1.00)$. However, the single-compartment illumination significantly decreased the activity level of all three groups in comparison to Trial 3 levels $(F=90.99, \mathrm{df}=1 / 32, \mathrm{p}<.01)$.

Analysis of the adaptation curves for successive 10-min periods of the $50-\mathrm{min}$ test indicated no significant difference in the trends of the three surgical groups under Trial 3 conditions of total darkness (Group by Successive Periods interaction, $F<1.00$ ) or under Trial 4 conditions of single-compartment illumination (Groups by Successive Periods interaction, F $<1.00$ ).

The mean percentage of total test time $(50 \mathrm{~min})$ spent in the illuminated compartment on Trial 4 by the normal-control, amygdala-sham, and amygdala-lesion groups was $7.49 \%, 8.40 \%$, and $3.19 \%$, respectively. Analysis of the data representing the percentage of total test time the animals spent in the illuminated compartment demonstrated no significant difference between the normal-control and amygdala-sham groups $(F<1.00)$. However, the amygdala-lesion group spent a significantly smaller percentage of total test time in the illuminated compartment than did the normal controls $(F=6.87, d f=1 / 21, p<.05)$ or the amygdala shams $(F$ $=4.70, \mathrm{df}=1 / 21, \mathrm{p}<.05)$.

Histological examination of the 12 amygdala-lesion brains indicated that all animals sustained bilateral damage to the corticomedial portion of the amygdala. In some instances, the damage also extended slightly posterior to the corticomedial amygdala and involved pre-hippocampal structures. A lesion representative of this group is reconstructed in Fig. 2. One animal (No. 31) had bilateral damage to cortical sites as well as to the amygdala due to electrode tract leakage. Since the brain damage and performance of this animal differed markedly from the brain damage and performance of other animals in the group, its scores were deleted from all previous summaries and analyses, with an appropriate adjustment being made in the statistical degrees of freedom. Histological examination of the 12 amygdala-sham brains indicated good bilateral 
placements and that the tips of the electrode tracts were generally in the same corticomedial region of the amygdala as were the lesions.

\section{DISCUSSION}

As measured in the present shuttlebox context (movement between compartments), the activity of amygdala-lesion animals does not differ significantly from that of either normal controls or amygdala shams. This conclusion holds true whether one considers the conditions of total apparatus darkness (Trial 3) or single-compartment illumination (Trial 4). Since activity in the present study did not include a measure of the movement of the animals within either compartment, a comparison of the results with the activity of rats in a moderately illuminated open-field test (Sclafani et al, 1970) or with the activity of rats in a highly illuminated open-field test (Schwartzbaum \& Gay, 1964) is difficult. In addition to major differences in the test situation, differences in the site and extent of amygdala damage further add to the difficulty of making such comparisons.

That the behavior of the animals in the present study was altered by the light in the illuminated compartment is clear from the fact that all groups spent less than $10 \%$ of the total test time in that area of the shuttlebox. Since the statistical analysis of Trials 1-3 rule out the contribution of apparatus novelty and unreliability of baseline activity to these findings, it seems clear that the illumination is the basic explanation for the reduction in the activity (movement between compartments) of all the groups on Trial 4. However, the amygdala-lesion animals demonstrated a significantly greater reactivity to light $(\mathrm{p}<.05)$ in the illuminated compartment than did the other two groups, which was independent of changes in level of activity; that is to say, they spent a significantly smaller percentage of total test time in the illuminated compartment, although they did not differ in the amount of movement between compartments. Such findings suggest that amygdala-lesion animals respond to the same environmental stimulus contingencies as do normal-control and amygdala-sham animals, but in the process of doing so, they may demonstrate a difference in their sensitivity to such conditions. Whether this difference in sensitivity is displayed or not experimentally may depend upon the specific nature of the task or behavior one is measuring. While the difference in the sensitivity of amygdala-lesion animals in the present study did not show up in the general activity data, the difference was apparent in the reactivity-to-light data.

The results of the present study support the view that the responsiveness of amygdala-lesion animals to

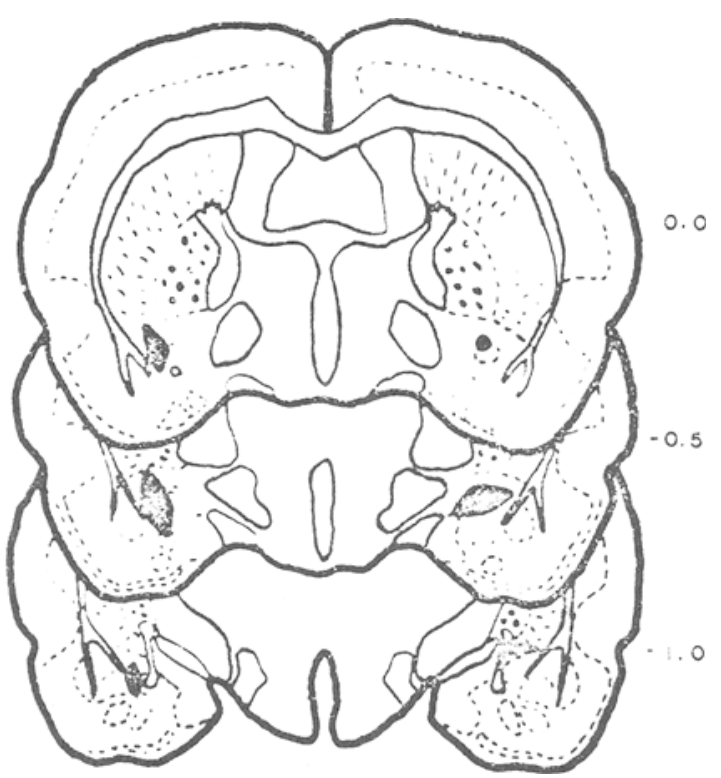

Fig. 2. Reconstruction of amygdala damage representative of that found in the lesion group (Animal 8). Reconstruction is adopted from Skinner (1971).

environmental stimuli may be altered, but that one should be cautious about the possible situational specificity of such a conclusion in terms of experimental tasks or requirements.

\section{REFERENCES}

Nauta, W. J. H. Central nervous organization and the endocrine motor system. In A. V. Nalbandov (Ed.), Advances in neuroendocrinology. Urbana: University of Illinois Press, 1963.

Schwartzbaum, J. S. Changes in reinforcing properties of stimuli following ablation of the amygdaloid complex in monkeys. Journal of Comparative \& Physiological Psychology, 1960, 53, 388-395.

Schwartzbaum, J. S. Discrimination behavior after amygdalectomy in monkeys: Visual and somesthetic learning and perceptual capacity. Journal of Comparative \& Physiological Psychology, 1965, 60, 314-319.

Schwartzbaum, J. S., \& Gay, P. E. Interacting behavioral effects of septal and amygdaloid lesions in the rat. Journal of Comparative \& Physiological Psychology, 1966, 61, 59-65.

Sclafani, A., Belluzzi, J. D., \& Grossman, S. P. Effects of lesions in the hypothalamus and amygdala on feeding behavior in the rat. Journal of Comparative \& Physiological Psychology, 1970, 72, 394-403.

Skinner, J. E. Neuroscience: A laboratory manual. Philadelphia: Saunders, 1971.

White, N. Perseveration by rats with amygdaloid lesions. Journal of Comparative \& Physiological Psychology. 1971, 77, 416-426.

(Received for publication February 5, 1973; revision received April 27, 1973: accepted May 15, 1973.) 